OPEN ACCESS

Edited by:

Anna Halama,

Weill Cornell Medicine-Qatar, Qatar

Reviewed by:

Philipp Antczak,

University Hospital of Cologne,

Germany

Stephen Atkin,

Royal College of Surgeons in Ireland,

Bahrain

${ }^{*}$ Correspondence:

Zhifu Wang

2007015@fjtcm.edu.cn

Jing Tao

taojing01@fitcm.edu.cn

Specialty section: This article was submitted to Systems Endocrinology, a section of the journal Frontiers in Endocrinology

Received: 10 November 2020 Accepted: 12 April 2021

Published: 10 June 2021

Citation:

Yu X, Chen X, Liu W, Jiang M, Wang Z and Tao J (2021) Proteomics Analysis

of the Spinal Dorsal Horn in Diabetic

Painful Neuropathy Rats With

Electroacupuncture Treatment.

Front. Endocrinol. 12:608183.

doi: 10.3389/fendo.2021.608183

\section{Proteomics Analysis of the Spinal Dorsal Horn in Diabetic Painful Neuropathy Rats With Electroacupuncture Treatment}

\author{
Xiangmei $\mathrm{Yu}^{1}$, Xiaomei Chen ${ }^{2}$, Weiting Liu $^{3}$, Menghong Jiang ${ }^{3}$, Zhifu Wang $^{1 *}$ \\ and Jing $\mathrm{TaO}^{2 *}$
}

${ }_{1}^{1}$ College of Integrated Traditional Chinese and Western Medicine, Fujian University of Traditional Chinese Medicine, Fuzhou, China, ${ }^{2}$ College of Rehabilitation Medicine, Fujian University of Traditional Chinese Medicine, Fuzhou, China, ${ }^{3}$ College of Acupuncture and Moxibustion, Fujian University of Traditional Chinese Medicine, Fuzhou, China

Background: Clinical evidence demonstrates that electro-acupuncture (EA) of the Zu sanli (ST36) and Shen shu (BL23) acupoints is effective in relieving diabetic painful neuropathy (DPN); however, the underlying molecular mechanism requires further investigation, including the protein molecules associated with EA's effects on DPN.

Methods: Sprague-Dawley adult male rats $(n=36)$ were randomly assigned into control, DPN, and EA groups ( $n=12$ each). After four weeks of EA treatment, response to mechanical pain and fasting blood glucose were analyzed. A tandem mass tag (TMT) labeling approach coupled with liquid chromatography with tandem mass spectrometry was used to identify potential biomarkers in the spinal dorsal horn. Further, proteomics analysis was used to quantify differentially expressed proteins (DEPs), and gene ontology, KEGG pathways, cluster, and string protein network interaction analyses conducted to explore the main protein targets of EA.

Results: Compared with the DPN model group, the mechanical pain threshold was significantly increased, while the fasting blood glucose levels were clearly decreased in EA group rats. Proteomics analysis was used to quantify 5393 proteins, and DEPs were chosen for further analyses, based on a threshold of 1.2-fold difference in expression level $(\mathrm{P}<0.05)$ compared with control groups. Relative to the control group, 169 downregulated and 474 up-regulated proteins were identified in the DPN group, while 107 and 328 proteins were up- and down-regulated in the EA treatment group compared with the DPN group. Bioinformatics analysis suggested that levels of proteins involved in oxidative stress injury regulation were dramatically altered during the EA effects on DPN.

Conclusions: Our results provide the valuable protein biomarkers, which facilitates unique mechanistic insights into the DPN pathogenesis and EA analgesic, antioxidant stress and hypoglycemic effect.

Keywords: proteomics, electroacupuncture, oxidative phosphorylation, neuropathic pain, diabetes 


\section{INTRODUCTION}

There are various types of chronic pain that can disturb the physical and mental health of individuals. Diabetic painful neuropathy (DPN) is a painful complication of diabetes, caused by constant high glucose levels. Approximately $20 \%$ of all diabetic patients present with neuropathic pain, manifesting as spontaneous pain, hyperalgesia, and allodynia (1). The streptozotocin (STZ)-diabetic rat model can be used to mimic the symptoms and pathological changes that occur during DPN. To date, the majority of studies have focused on peripheral sensory nerve function; however, the spinal cord is also important in the development of DPN (2). A number of morphological studies have demonstrated spinal cord damage and atrophy in patients with diabetes, and peripheral nerve stimulation can reduce blood-oxygen level-dependent activity in the dorsal horn of diabetic rats $(3,4)$.

Electroacupuncture (EA), as a beneficial acupuncture therapy, is effective in alleviating pain and improving quality of life in patients with diabetic peripheral neuropathy (5-7). Zusanli (ST36) and Shenshu (BL23) are common and effective acupoints used for treatment of diabetic peripheral neuropathy (8-10).

Proteomics analysis provides a valuable strategy for exploring the pathogenesis of diabetes mellitus, as well as therapeutic targets in this condition. Some proteomic studies have been performed in animal models of DPN or diabetic neuropathy, to search for differentially expressed proteins (DEPs) induced by STZ. Mitochondrial oxidative phosphorylation, metabolic dysregulation of mitochondrial complexes, and antioxidases are involved in DPN progression and represent potentially important therapeutic targets. However, previous studies have focused only on the proteomes of the sciatic nerve, lumbar $4 / 5$ dorsal root ganglia, and trigeminal ganglia, while the exact spinal mechanism underlying DPN is far from clear and additional potential spinal biomarkers are needed (11-14).

\section{MATERIALS AND METHODS}

\section{Animals and Ethics Statement}

Male Sprague-Dawley rats weighing $160 \pm 20 \mathrm{~g}$ were obtained from the Experimental Animal Center of Fujian University of Chinese Medicine. Rats were housed under standard living conditions at $22^{\circ} \mathrm{C}$ and a $12 \mathrm{~h} \mathrm{light/dark}$ cycle with sufficient food and water and acclimatized to the pain-testing environment before the experiments. All animal procedures were in strict accordance with relevant international laboratory animal use guidelines. After adaptive feeding, rats were randomly allocated into three groups as follows: Control group $(n=12)$, DPN group $(n=12)$, and EA group $(n=12)$.

According to the previous literature reports, diabetes was induced by a single intraperitoneal injection of STZ $(65 \mathrm{mg} / \mathrm{kg}$, Sigma Chemicals, USA) dissolved in citrate buffer $(10 \mathrm{mmol} / \mathrm{L}$, $\mathrm{Na}$ citrate, $\mathrm{pH}=4.3$ ) (15). The control group received an equivalent volume of citrate buffer only. Two weeks later, tail venous fasting blood glucose (FBG) were measured using a glucometer (OEM, USA). Only rats with FBG $>16.7 \mathrm{mmol} / \mathrm{L}$ and mechanical paw withdrawal threshold (PWT) $<5 \mathrm{~g}$ were included in further experiments.

All experimental procedures and protocols were approved by the Animal Research and Ethics Committee of Fujian University of Traditional Chinese Medicine. At the endpoint of the experiment, all rats were euthanized, according to the Care Guidelines.

\section{Electroacupuncture (EA) Procedure}

In the EA group, the rats were loosely fixed on a wooden stand, where their head and limbs could move freely. After the rats were stabilized, the acupoints "Zu sanli" (ST36) and "Shen shu" (BL23) were selected for acupuncture and electrical stimulation (Figure 1A). EA intervention began at two weeks after STZ injection. ST36 is located at the posterolateral knee about $5 \mathrm{~mm}$ below the fibula head (16), while BL23 is located in the depressions lateral to the lower border of the spinous processes of the second lumbar vertebrae, approximately $8 \mathrm{~mm}$ from the midline of the adult rat (17). Needles inserted at ST36 and BL23 were connected to a G6805-1A multifunctional EA apparatus (Shanghai Medical Electronic Apparatus Company, Shanghai, China), with a stimulation intensity of $1 \mathrm{~mA}$, frequency $10 \mathrm{HZ}$. According to previous literature reports, EA stimulation was maintained for $30 \mathrm{~min}$ once every other day for a consecutive four weeks $(15,18)$.

\section{Measurement of Paw Withdrawal Threshold}

The mechanical PWT was examined before and after injection of STZ or citrate buffer $(0,2,6$ weeks after injection). The test environment was a $10 \mathrm{~cm} \times 20 \mathrm{~cm} \times 20 \mathrm{~cm}$ plastic cage with a plexiglass floor. After the rats were placed in the cage and allowed to stand for 10 minutes, the center of the feet of the rats were stimulated with different tense by von Frey Hairs (0.4, 0.6, 1.4, 2, $4,6,8,10$, and 15g) according to the up-down method described by Dixon (19). Each von Frey hair was held for about 1-2 s, with 10min intervals between each stimulation. Testing was initiated with the $2.0 \mathrm{~g}$ hair.

The positive response was defined as a withdrawal of hind paw upon the stimulus. Whenever a positive response appeared, the next lower gram was applied, and whenever a negative response occurred, the next higher gram was applied. The testing consisted of five more stimuli after the first change in response occurred, and the pattern of response was converted to a 50\% von Frey threshold using the method described by Dixon and based on our previous literature $(20,21)$. All data are presented as mean \pm SE.

\section{Sample Collection}

To investigate DEPs in three groups, all rats were sacrificed under low dose isoflurane $(0.5-1 \%)$ anesthesia, six weeks after STZ injection. The $\mathrm{L}_{4-5}$ spinal cords were rapidly dissected and immediately placed in $-80^{\circ} \mathrm{C}$ refrigerator for the further proteomics detection. 
A
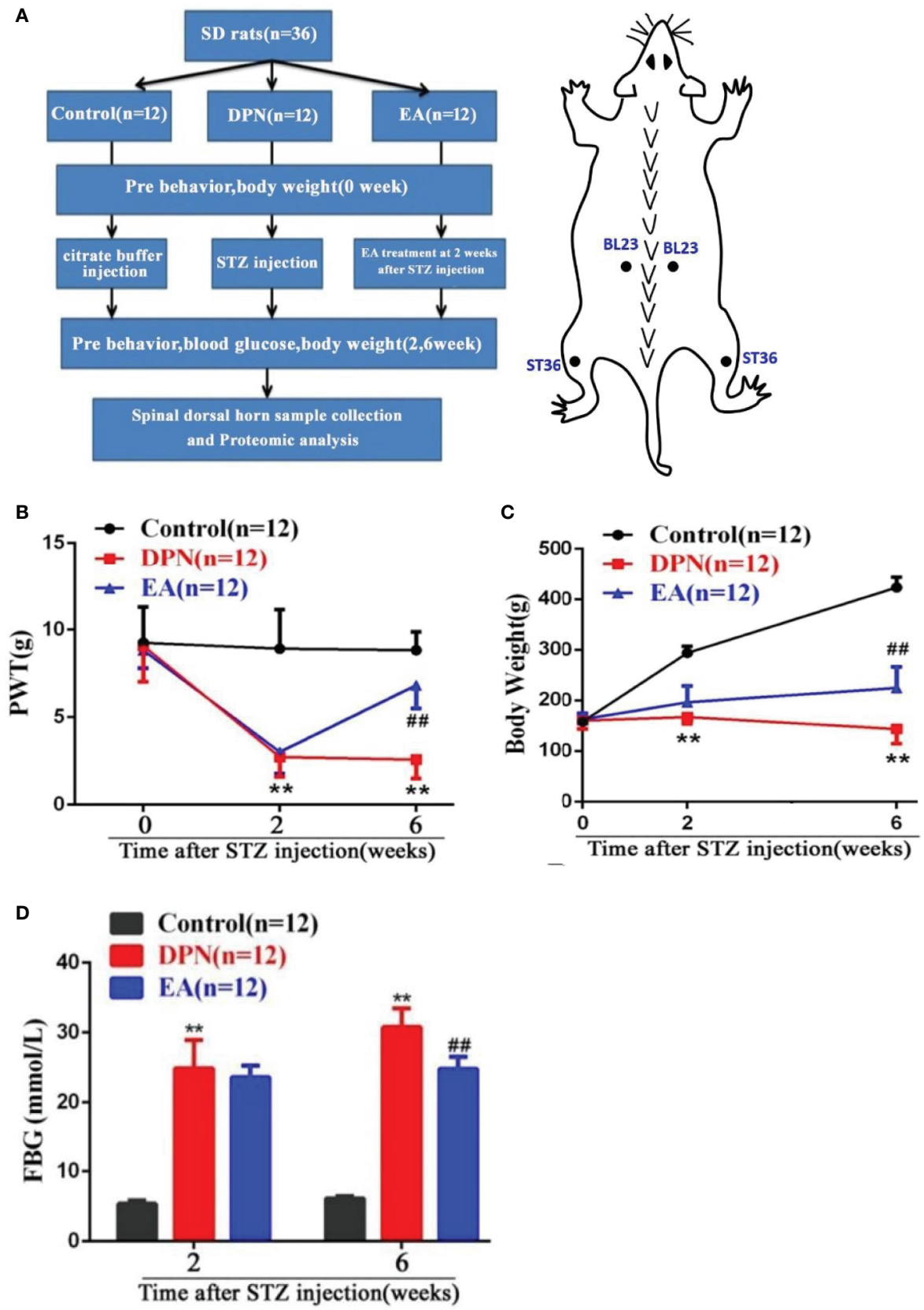

FIGURE 1 | Flow chart of the experiment and the changes of body weight, paw withdrawal threshold (PWT) and fast blood glucose (FBG) in DPN rats. (A) Flow chart of the experiment. (B) Body weight variation in Control, DPN, and EA groups rats. Two-way repeated ANOVA, F2, 33 = 237.4, P<0.001; post hoc Turkey test: DPN compared with Control $\left({ }^{\star *} P<0.01\right)$, EA compared with DPN (\#\# $\left.P<0.01\right)$. (C) Significant changes of PWT in different groups. Two-way repeated ANOVA, F2, $33=59.608, P<0.001$; post hoc Turkey test: DPN compared with Control $\left({ }^{\star \star} P<0.01\right)$, EA compared with DPN $\left({ }^{\# \# ~} P<0.01\right)$. (D) Significant changes of FBG in different groups. Two-way repeated ANOVA, F1, $33=299.3, P<0.001$; post hoc Turkey test: DPN compared with Control ( $\left.{ }^{\star \star} P<0.01\right)$, EA compared with DPN $(\# \#<0.01)$.

\section{Proteomics Experiment and Proteomics Data Analysis}

\section{Protein Extraction and Trypsin Digestion}

The $\mathrm{L}_{4-5}$ spinal cord tissue samples were removed from $-80^{\circ} \mathrm{C}$ freezer, and an appropriate amount of sample was added to a pre-chilled mortar with liquid nitrogen and ground to powder. Proteins were then extracted from samples in lysis buffer ( $8 \mathrm{M}$ urea, $1 \%$ protease inhibitor) using a high-intensity ultrasonic processor. Remaining debris was removed by centrifugation at $12,000 \mathrm{~g}$ for $10 \mathrm{~min}$ at $4^{\circ} \mathrm{C}$. The protein concentration of the supernatant was detected with BAC kits. 
For trypsin digestion, protein solution was reduced with dithiothreitol (final concentration, $5 \mathrm{mM}$ ) for $30 \mathrm{~min}$ at $56^{\circ} \mathrm{C}$, then incubated with iodoacetamide (final concentration, $11 \mathrm{mM}$ ) for $15 \mathrm{~min}$ at $37^{\circ} \mathrm{C}$ in darkness. Then, the protein samples were diluted with $\mathrm{mM}$ triethylamonium bicarbonate (TEAB), until the urea concentration was $<2 \mathrm{M}$. Finally, trypsin was added at a mass ratio (trypsin: protein) of 1:50 for the first digestion at $37^{\circ} \mathrm{C}$ overnight, then of $1: 100$ for the second digestion (4h, $\left.37^{\circ} \mathrm{C}\right)$.

\section{TMT Labeling}

After trypsin digestion, peptides were desalted and vacuum-dried with the strata X C18 SPE column. Peptides were reconstituted in $0.5 \mathrm{M}$ TEAB and marked according to the TMT kit protocols.

\section{LC-MS/MS Analysis}

Separated peptides were subjected to sodium/iodide symporter sources. Tandem mass spectrometry (MS/MS) was performed using a Q ExactiveTM Plus (Thermo) instrument, coupled online to an ultra-performance liquid chromatography system.

Electrospray voltage was $2.0 \mathrm{kV}$, and intact peptides were detected in the Orbitrap at 70,000 mass resolution. The primary MS scan range was $350-1600 \mathrm{~m} / \mathrm{z}$. Collected data were processed using a data-dependent scanning program (DDA). Automatic gain control was set at 5E4, signal threshold as 5000 ions/s, maximum time $200 \mathrm{~s}$, and dynamic exclusion time of the tandem mass scan $15 \mathrm{~s}$, to avoid repeated scanning of precursor ions.

\section{Database Search}

MS/MS data were analyzed using the max quant search engine (v.1.5.2.8), with parameters as follows: Rat_Uniprot was first screened, then a reverse library added to calculate the false positive ratio (FPR); Trypsin/P was specified as the cleavage enzyme, allowing up to two missing cleavages; minimum peptide length was seven amino acid residues; maximum number of peptide modifications was five; mass tolerance for the primary precursor ions search was $20 \mathrm{ppm}$ and $5 \mathrm{ppm}$ for the main search; mass tolerance for fragment ions was $0.02 \mathrm{Da}$; the quantitative method was set to TMT-10plex; and the FPR for peptide-spectrum match identification was $1 \%$.

\section{Bioinformatics Annotation}

Gene Ontology (GO) annotations were derived from the UniProtGOA database (http://www.ebi.ac.uk/GOA/). InterProScansoft was used to elucidate the GO categories: molecular function, cellular component, and biological process. KEGG online service tools were used to annotate protein descriptions, which were matched into corresponding pathways using KEGG mapper. Wolfpsort (https://wolfpsort.hgc.jp/) was used to investigate subcellular localization. KEGG database pathway enrichment analysis was conducted using a two-tailed Fisher's exact test. Pathways were classified according to the KEGG website.

Further cluster analysis of function enrichment was conducted to explore potential connections and differences in specific functions. First, all categories were collected after enrichment, according to their $\mathrm{P}$ values, then filtered for those categories with $\mathrm{P}$ value $<0.05$. This filtered $\mathrm{P}$ value matrix was transformed using the function $\mathrm{x}=-\log 10$ ( $\mathrm{P}$ value). Finally, these $\mathrm{x}$ values were $\mathrm{z}$-transformed for each functional category. Cluster membership was visualized using a heat map.

\section{Protein-Protein Interaction Network}

Numbers or sequences of DEPs were compared using the STRING (v.10.5) protein network interaction database. An interaction relationship was extracted according to a confidence score $>0.7$ (high confidence).

\section{Statistical Analysis}

All data are presented as the mean \pm standard deviation (SD). The three groups were analyzed by the Analysis Of Variance (ANOVA) and post hoc Tukey test in the SPSS 21.0 software (SPSS, Armonk, NY, USA). Graphs were generated by using GraphPad Prism 7.0 software.

\section{RESULTS}

\section{Electroacupuncture Significantly Reduced Mechanical Hypersensitivity and Fasting Blood Glucose Levels During the Development of DPN.}

As shown in the flow chart of the experiment and acupoint diagram (Figure 1A), DPN model of rats and EA intervention were established and fasting blood glucose and behavioral tests conducted before and after STZ injection and EA intervention. Compared with the control group, body weight and mechanical pain threshold were significantly reduced while fasting blood glucose was dramatically increased at 2 weeks after STZ injection $\left.{ }^{* *} P<0.01\right)$.

However, compared with the DPN model group, the body weight and mechanical pain threshold were obviously upgraded, while the fasting blood glucose was soothingly downgraded after four weeks of EA treatment $(\# \# P<0.01)$ (Figures 1B-D).

\section{Quantitative of Differentially Expressed Proteins}

A total 5744 proteins were identified from spinal dorsal horn samples from the three experimental groups, and 5393 proteins were quantified after proteomic analysis. Molecules with expression ratios showing a $>1.2$-fold change and $\mathrm{p}$-values < 0.05 were considered DEPs.

Compared with the control group, levels of 435 proteins were significantly changed (328 down- and 107 up-regulated) in DPN rats and 643 proteins changed in EA rats $(\mathrm{p}<0.05$ and foldchange $>1.5$ ) (Figure 2A). Further analysis showed that 118 proteins were significantly changed (25 down- and 93 upregulated) in DPN rats while 14 proteins were up-regulated and 83 proteins down-regulated after EA treatment $(\mathrm{p}<0.05$ and fold-change $>1.5$ ) (Figure 2B). There were 51 common positive proteins when DPN up-regulated and EA downregulated $(\mathrm{p}<0.05$ and fold-change $>1.5$ ), while 8 common positive proteins were found which DPN down-regulated and EA-upregulated $(\mathrm{p}<0.05$ and fold-change $>1.5$ ). There were 


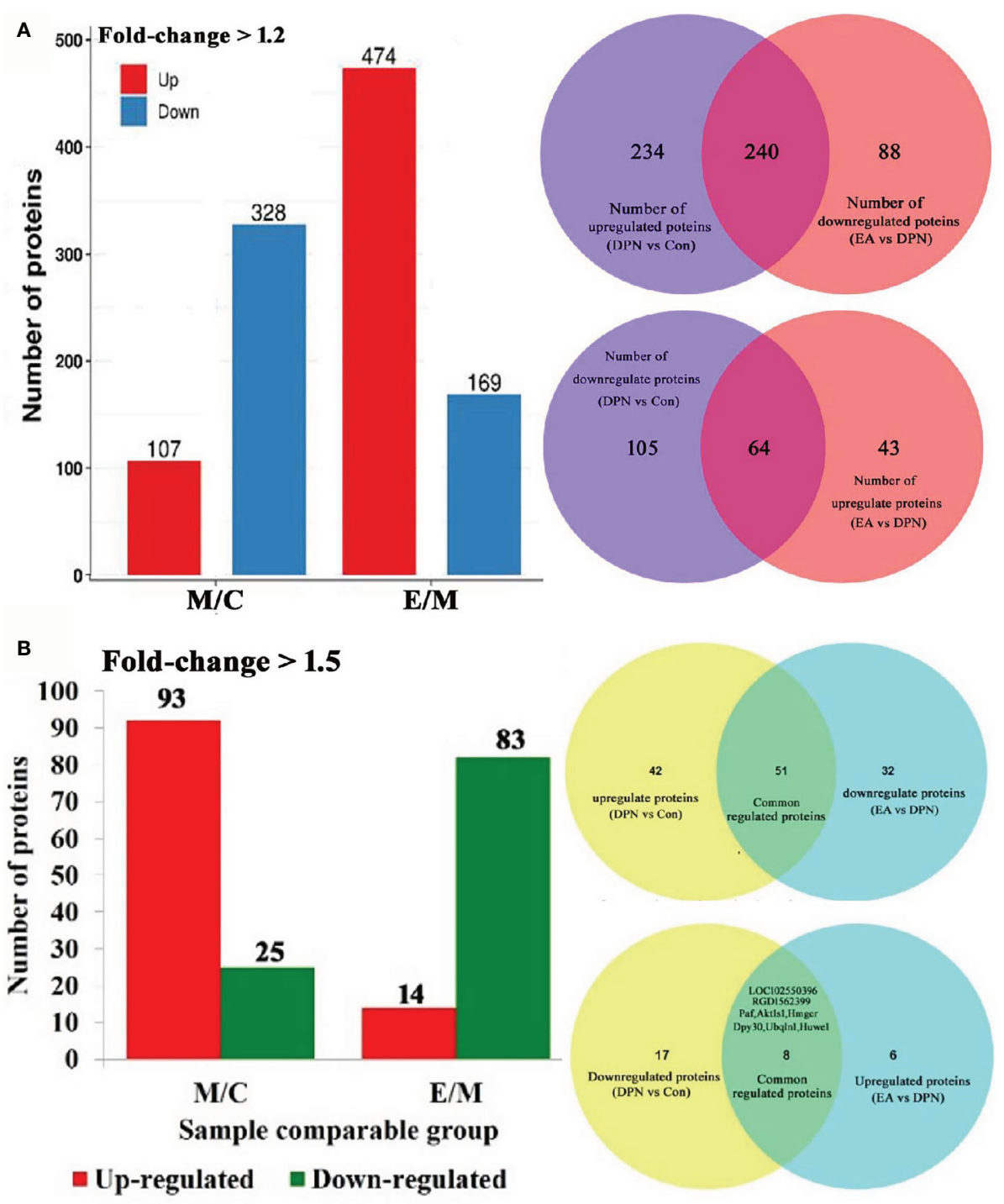

FIGURE 2 | Quantitative of differentially expressed proteins in three groups. (A) Number of differential protein set at 1.2-fold (M/C, DPN group compared with control group E/M; EA group compared with DPN group). 240 common positive proteins when DPN up-regulated and EA down-regulated set at 1.5-fold. 64 common positive proteins when DPN down-regulated and EA up-regulated set at 1.5-fold. (B) Number of differential protein set at 1.5-fold (M/C, DPN group compared with control group E/M; EA group compared with DPN group). 51 common positive proteins when DPN up-regulated and EA down-regulated set at 1.5-fold. 8 common positive proteins when DPN down-regulated and EA up-regulated set at 1.5-fold.

total 59 common changed proteins shown in the Table $\mathbf{1}$ ( $\mathrm{p}<0.05$ and fold-change $>1.5$ ).

\section{Molecular Function, Cellular Component, and Biological Process in Control, DPN, and EA Group Rats}

GO analysis was used to evaluate the molecular function, cellular component, and biological processes enriched for DEPs.

In terms of biological processes, the top five enriched for DPNassociated proteins were cellular process, single-organism process, biological regulation, metabolic process, and response to stimulus, while the top five enriched for EA-regulated proteins were single- organism process, cellular process, metabolic process, biological regulation, and response to stimulus. Cell component analysis indicated the most enriched for DPN-associated proteins were cell, organelle, membrane, extracellular region, and macromolecular complex, among others, while EA-regulated proteins were mainly involved in the cell, organelle, membrane, macromolecular complex, and extracellular region. Molecular functions analysis suggested that the most common molecular functions of DPNassociated and EA-regulated proteins were binding and catalytic activity (Figures 3A, B).

According to subcellular structural localization analysis, the main subcategories $(>10 \%)$ in DPN-associated proteins were 
TABLE 1 | The common significant differences of EA-regulated and DPN-associated proteins $(p<0.05$ and changes $>1.5$-fold).

\begin{tabular}{|c|c|c|c|c|c|c|}
\hline Protein accession & Protein description & Gene name & DPN/CON Ratio & $P$ value & EA/DPN Ratio & $P$ value \\
\hline F7F9X9 & - & Slc26a8 & 14.35 & $2.28 \mathrm{E}-06$ & 0.076 & 1.61E-05 \\
\hline D3ZUF8 & - & Vrk2 & 4.899 & 3.56E-05 & 0.216 & 0.000162 \\
\hline F7EUK4 & T-kininogen 2 & Kng111 & 4.212 & 2.17E-05 & 0.449 & 4.18E-05 \\
\hline Q5YIR9 & - & Clec4a1 & 3.793 & 1.69E-08 & 0.225 & 0.001378 \\
\hline P17712 & Glucokinase & Gck & 3.655 & 6.97E-07 & 0.311 & 0.003456 \\
\hline P01048 & T-kininogen 1 & Map1 & 3.457 & $3.28 \mathrm{E}-06$ & 0.528 & 4.31E-05 \\
\hline $\mathrm{AOAOH} 2 \mathrm{UHM} 3$ & Haptoglobin & $\mathrm{Hp}$ & 2.313 & 0.035243 & 0.451 & 0.000338 \\
\hline MORAVO & - & - & 2.242 & 0.000317 & 0.646 & 0.001018 \\
\hline F1M5A4 & Katanin p60 ATPase-containing subunit A-like 2 & Katnal2 & 2.238 & $1.69 \mathrm{E}-05$ & 0.48 & $2.22 \mathrm{E}-05$ \\
\hline MOR4Z4 & - & LOC682352 & 2.207 & 4.27E-05 & 0.636 & 0.000698 \\
\hline P20767 & Ig lambda-2 chain $\mathrm{C}$ region & - & 1.995 & 0.013403 & 0.642 & 5.97E-05 \\
\hline G3V9P3 & - & MGC112715 & 1.95 & 2.46E-05 & 0.499 & 3.88E-05 \\
\hline D3ZNA5 & Class I histocompatibility antigen, Non-RT1.A alpha- 1 chain & LOC686860 & 1.878 & 4.33E-05 & 0.519 & 4.1E-05 \\
\hline B1WBQ8 & Glyceraldehyde-3-phosphate dehydrogenase & Gapdhs & 1.848 & 0.007075 & 0.626 & 0.001681 \\
\hline Q7TQ70 & Fibrinogen alpha chain & Fga & 1.823 & 1.17E-06 & 0.647 & 3.94E-06 \\
\hline D3ZV51 & Olfactory receptor & LOC100909611 & 1.822 & 0.010757 & 0.427 & 0.000603 \\
\hline D4A6F2 & - & Lynx1 & 1.822 & 0.000436 & 0.605 & 0.000517 \\
\hline A0A0G2KB63 & Prohibitin-2 & Phb2 & 1.821 & 3.07E-06 & 0.55 & 4.52E-06 \\
\hline P02680 & Fibrinogen gamma chain & Fgg & 1.796 & 4.31E-05 & 0.664 & 0.000184 \\
\hline P14480 & Fibrinogen beta chain & Fgb & 1.789 & $1.58 \mathrm{E}-05$ & 0.644 & 1.64E-05 \\
\hline P67779 & Prohibitin & $\mathrm{Phb}$ & 1.751 & 3.53E-05 & 0.555 & 0.000384 \\
\hline F1M4J0 & - & Rictor & 1.748 & 0.00012 & 0.59 & 9.54E-05 \\
\hline A0A096MJ90 & - & Ly6h & 1.713 & $8.48 \mathrm{E}-05$ & 0.529 & 6.06E-05 \\
\hline Q7TPI6 & - & rCG_62991 & 1.69 & 0.001398 & 0.643 & 0.010803 \\
\hline Q66HL0 & 5-nucleotidase & $\mathrm{N}+5 \mathrm{e}$ & 1.689 & 0.003575 & 0.644 & 0.008803 \\
\hline P11951 & Cytochrome c oxidase subunit 6C-2 & Cox6c2 & 1.647 & $1.33 E-06$ & 0.495 & 4.16E-05 \\
\hline G3V851 & - & Slc17a6 & 1.64 & 0.001939 & 0.608 & 9.99E-05 \\
\hline P80431 & Cytochrome c oxidase subunit 7B, mitochondrial & Cox7b & 1.634 & $1.48 \mathrm{E}-06$ & 0.537 & 8.1E-07 \\
\hline BOBNE6 & - & Ndufs8 & 1.62 & 0.000135 & 0.647 & 0.000305 \\
\hline P01836 & Ig kappa chain $\mathrm{C}$ region, $\mathrm{A}$ allele & - & 1.597 & 0.002182 & 0.362 & 0.000221 \\
\hline P10888 & Cytochrome c oxidase subunit 4 isoform 1 , mitochondrial & Cox4i1 & 1.574 & 4.74E-06 & 0.573 & $1.62 \mathrm{E}-06$ \\
\hline P11661 & $\mathrm{NADH}$-ubiquinone oxidoreductase chain 5 & Mtnd5 & 1.572 & 0.001844 & 0.597 & 5.64E-05 \\
\hline P80432 & Cytochrome c oxidase subunit 7C, mitochondrial & Cox7c & 1.562 & 0.000196 & 0.5 & 0.000103 \\
\hline D3ZLT1 & - & Ndufb7 & 1.559 & $5.66 \mathrm{E}-05$ & 0.585 & $8.2 E-05$ \\
\hline D4A565 & - & Ndufb5 & 1.557 & $2.11 \mathrm{E}-05$ & 0.582 & 4.47E-06 \\
\hline F1LXA0 & - & Ndufa12 & 1.555 & $1.88 \mathrm{E}-05$ & 0.625 & 3.16E-06 \\
\hline D3ZYX8 & - & Cox7a2l & 1.547 & 0.000164 & 0.631 & 0.000143 \\
\hline F1LTP5 & Coiled-coil domain-containing protein 63 & Ccdc63 & 1.546 & $8.42 \mathrm{E}-05$ & 0.622 & 0.00018 \\
\hline A0A0G2K7D4 & Dynein heavy chain 12 , axonemal & Dnah12 & 1.545 & $2.08 \mathrm{E}-05$ & 0.611 & 0.000599 \\
\hline Q5M7T6 & - & Atp6v0d1 & 1.545 & 3.57E-05 & 0.58 & 4.5E-05 \\
\hline D4ACP8 & - & Serac1 & 1.542 & 0.000463 & 0.664 & 0.00038 \\
\hline D4A463 & - & Tpgs1 & 1.535 & 0.024738 & 0.579 & 0.006541 \\
\hline D3ZG43 & - & Ndufs3 & 1.521 & $1.75 \mathrm{E}-05$ & 0.641 & 1.69E-06 \\
\hline A0A2U3UXS5 & - & $\operatorname{lgsf21}$ & 1.519 & 0.006782 & 0.583 & 0.00152 \\
\hline $\mathrm{AOAOH} 2 \mathrm{UHV} 2$ & Mediator of RNA polymerase II transcription subunit 23 & Med23 & 1.514 & 0.000217 & 0.666 & 0.000244 \\
\hline F1M0G0 & - & Rgs17 & 1.51 & 0.006183 & 0.579 & 0.001856 \\
\hline P12075 & Cytochrome c oxidase subunit 5B, mitochondrial & Cox5b & 1.507 & 3.79E-05 & 0.587 & 4.56E-06 \\
\hline F1M9G7 & CREB-binding protein & Crebbp & 1.506 & 0.00094 & 0.623 & $6.26 \mathrm{E}-05$ \\
\hline B0BN66 & - & Sapcd2 & 1.505 & 0.005243 & 0.635 & 0.003216 \\
\hline B5DEL8 & - & LOC100363268 & 1.504 & 0.000462 & 0.663 & 0.000245 \\
\hline F1LPG5 & - & LOC688963 & 1.5 & 6.33E-05 & 0.637 & 0.000138 \\
\hline Protein accession & Protein description & Gene name & DPN/CON Ratio & $P$ value & EA/DPN Ratio & $P$ value \\
\hline A0A0G2JUM9 & - & LOC102550396 & 0.366 & 2.4303E-05 & 2.492 & 0.0001 \\
\hline D3ZAS1 & - & RGD1562399 & 0.419 & 3.5258E-05 & 2.183 & 0.000136 \\
\hline Q6RIA2 & PCNA-associated factor & Paf & 0.468 & 5.7355E-05 & 1.548 & 0.001543 \\
\hline D3ZH75 & - & Akt1s1 & 0.525 & 0.0183551 & & \\
\hline P51639 & 3-hydroxy-3-methylglutaryl-coenzyme A reductase & Hmgcr & 0.601 & 0.0025202 & 1.573 & 0.007535 \\
\hline G3V946 & - & Dpy30 & 0.639 & 0.0141759 & 1.757 & 0.005797 \\
\hline A0A140TAl1 & - & Ubqln1 & 0.647 & 4.0053E-05 & 1.54 & 0.000103 \\
\hline P51593 & E3 ubiquitin-protein ligase HUWE1 & Huwe1 & 0.665 & 5.9014E-05 & 1.543 & 0.00098 \\
\hline
\end{tabular}

cytoplasm (26.59\%), extracellular (21.46\%), nucleus (18.35\%), plasma membrane (14.31\%), and mitochondria (10.42\%). The same analysis of EA-associated proteins also identified the cytoplasm (27.36\%), extracellular (18.62\%), nucleus (17.47\%), plasma membrane (15.4\%), and mitochondria (13.56\%) subcategories (Figures 3C, D). 

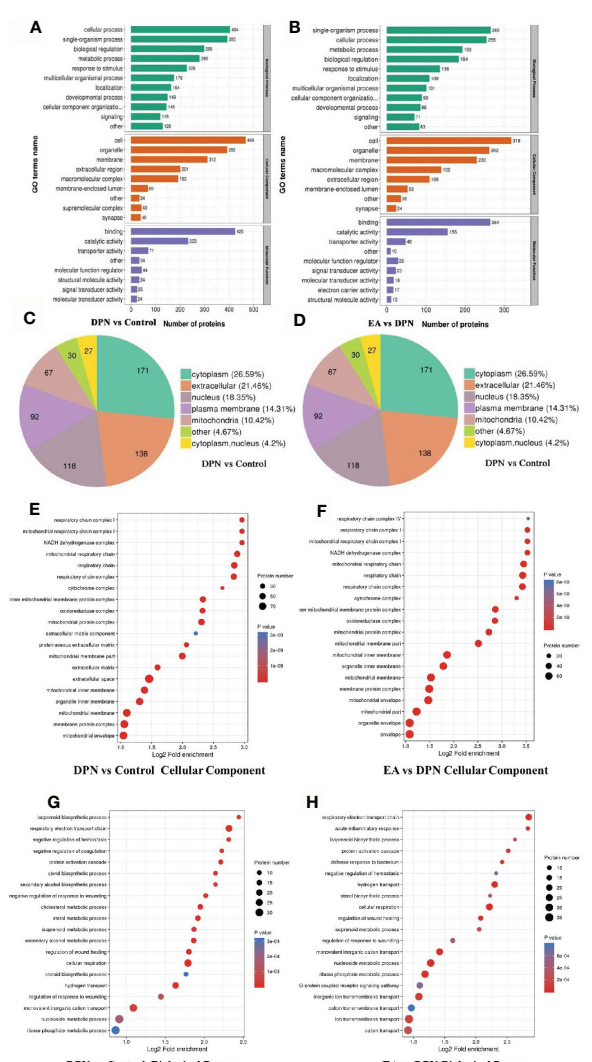

H

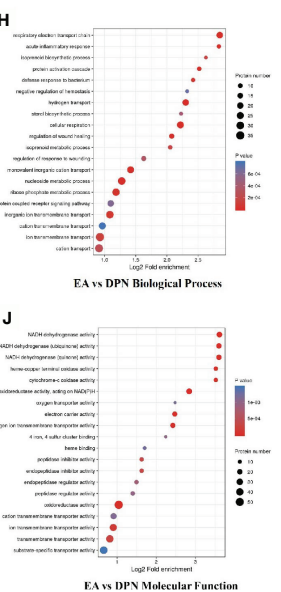

FIGURE 3 | GO annotation analysis showed the potential biomarkers mainly involved in DPN pathology and EA treatment (A) GO terms name of molecular function, cellular component, and biological process in DPN-associated proteins (B) GO terms name of molecular function, cellular component, and biological process in EAregulated proteins (C) Subcellular structural localization analysis in DPN-associated proteins (D) Subcellular structural localization analysis in EA-regulated proteins.

(E-J) GO annotation summarizing in cellular component, molecular function and biological process in different compared groups.

Last, GO analysis demonstrated that the cellular component, biological process molecular function of DPN and EA treatment were closely related to the mitochondrial respiratory chain and NADH dehydrogenase activity (Figures 3E-J).

\section{Functional Enrichment Analysis With KEGG Pathway and Cluster Analysis of KEGG Pathway Functional Correlation of the DEPs}

To further explore pathways significantly enriched for DEPs (DPN vs. Control, $\mathrm{n}=643$; EA vs. DPN, $\mathrm{n}=435$ ), DEPs were mapped to the KEGG database and enrichment levels calculated (Fisher's exact test; -log 10[p-value]), where a higher p-value indicates more DEPs enriched in a category. As shown in Figures 4A, B, KEGG pathway enrichment analysis demonstrated that DPN-associated and EA-regulated DEPs were mainly enriched in several pathways, particularly oxidative phosphorylation (Figure 4C). Further, remarkable changes were observed in ten proteins closely related to oxidative phosphorylation during EA treatment of DPN rats (Table 2 and Figure 4D).

\section{DISCUSSION}

In our research, we found that four weeks EA can improve weight loss, blood glucose, and sensitivity to mechanical pain in DPN rats. We generated proteomics data from DPN rat spinal dorsal horn samples treated with repeated EA stimulation. A total of 5393 proteins were quantified using the TMT labeling proteomics method. Of these proteins, 93 were down-regulated, 


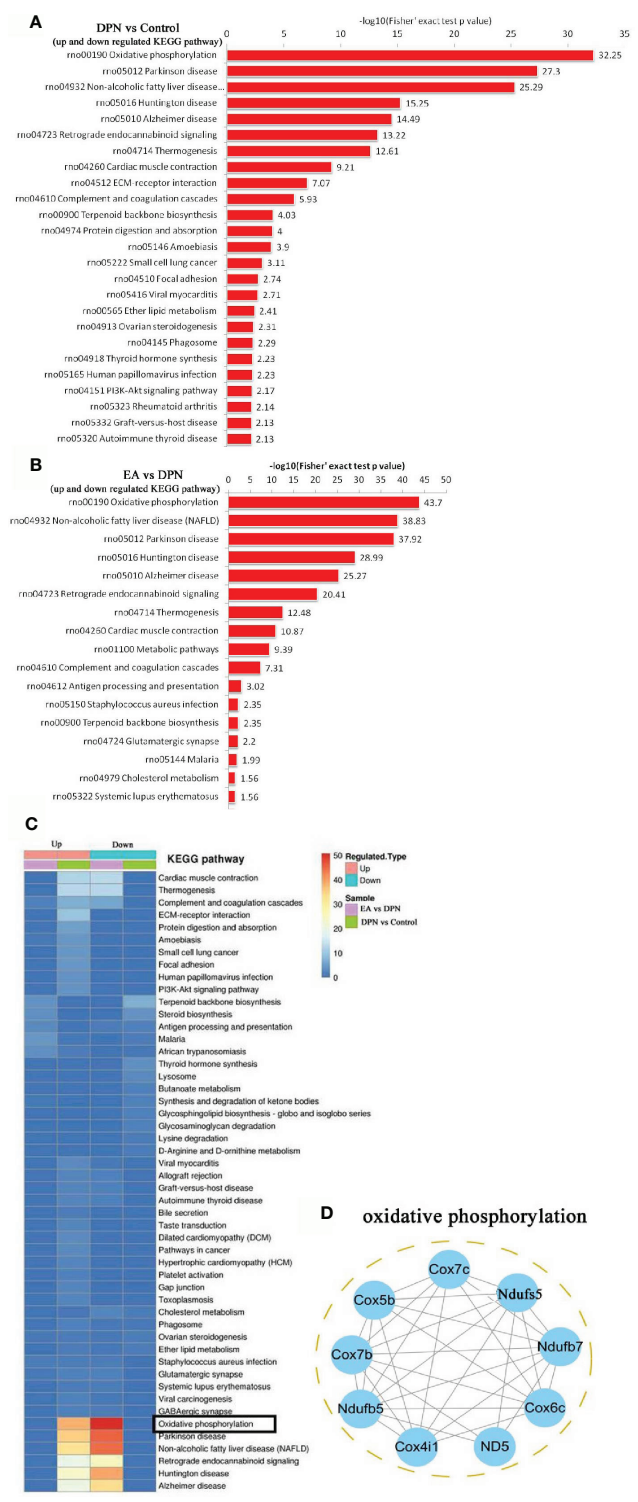

FIGURE 4 | KEGG pathway and related oxidative phosphorylated proteins analysis (A) the KEGG pathway enrichment analysis in DPN rats compared with control rats. (B) The KEGG pathway enrichment analysis in EA group rats compared with DPN rats. (C) The KEGG pathway analysis in different group rats. (D) Networks Analysis of major proteins in oxidative phosphorylation.

TABLE 2 | The prominent changes of oxidative phosphorylated proteins during EA treatment with DPN rat.

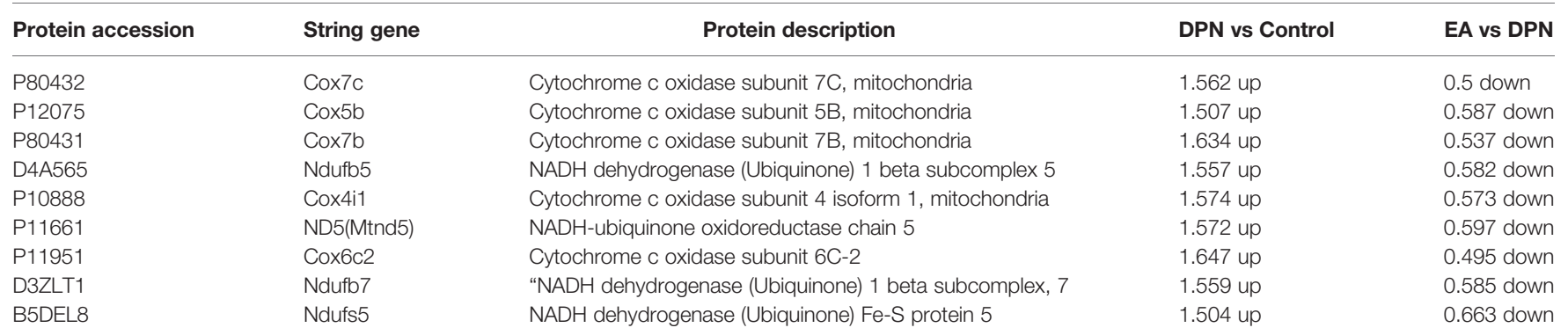


while 25were up-regulated during the development of DPN. After EA stimulation intervention, 14 up-regulated and downregulated 83 proteins were detected in the spinal dorsal horn. KEGG pathway enrichment analysis suggested that oxidative phosphorylation was a major factor involved in the effects of EA therapy on DPN. The results of this study provide a potentially valuable theoretical underpinning of the mechanisms underlying DPN pathophysiology and the clinical application of acupuncture.

The occurrence of diabetes and its complications are closely related to the mechanism of oxidative phosphorylation via the disturbances of mitochondrial ATP, complex IV and reactive oxygen species (ROS) metabolism (22-24). Upregulated expression of complexes I, III, IV, and V was found in the sciatic nerve of diabetic neuropathy rats (11). In addition, mitochondrial complex III of dorsal root ganglia was significantly decreased, while glutathione peroxidase and peroxidase were increased during STZ induced diabetic peripheral neuropathy (13).

Our results indicate that cytochrome c oxidase (Cox, Complex IV) factors, including Cox4i1, Cox 5b, Cox6c2, Cox7b, and Cox7c, were significantly upregulated in spinal dorsal horn during DPN, as were NADH-ubiquinone oxidoreductase (Complex I) factors, including Ndufb5, Ndufb7, andNdufs5. Further, treatment with EA could decrease the up-regulation of $\mathrm{Cox}$ and $\mathrm{NADH}$ oxidoreductase. As we know, abnormal function of mitochondrial Cox and $\mathrm{NADH}$ oxidoreductase could induce ROS overproduction, caspase 3 activation cell apoptosis $(25,26)$. Spinal ROS overproduction and activated caspase- 3 induced by Cox play a critical role in microglial activation and neuronal apoptosis in several neuropathic pain models included DPN $(27,28)$. Blocking NADPH oxidase, scavenging ROS, and reducing oxidative phosphorylation by various pharmacotherapy could prevent the induction of spinal long-term potentiation and relieve the persistent pain (29-31).

In addition, EA can also inhibit oxidative phosphorylation and ROS production through different pathways in neuropathic pain and other chronic diseases. For example,

Neuronal ATP receptor P2X7 activation leads to ROS production and subsequent nociceptive pain $(32,33)$. EA can improve neuropathic pain by inhibiting oxidative phosphorylation, reducing ATP production, and suppressing central sensitization in the spinal dorsal horn (34). In middle cerebral artery occlusion rats, EA treatment could significantly improve the respiratory control ratio, and increase the activities of NADH dehydrogenase and Cox in the perifocal penumbra zone (35). In the state of ischemia reperfusion injury, EA can inhibit cardiac Cox and indirectly inhibit the activation of caspase 3 (36). However, EA can alleviate bupivacaine-induced myocardial injury by upregulating enzymatic activity of Cox (37). In a word, EA can biaxially regulate oxidative phosphorylation of Cox and NADH dehydrogenase with bidirectional properties in different diseases and tissues.

According to the above research reports, combined with our results, we speculated that EA can reduce diabetic neuropathic pain by downregulating Cox and NADH dehydrogenase in the spinal cord, which may mainly be through the following ways: (1) inhibiting the spinal ATP release and P2X7 activation, reducing the spinal neuronal abnormal excitability and microglial activation; (2) reducing spinal ROS production and oxidative phosphorylation injury, inhibiting cell injury and even apoptosis. In the next experiment, we will further explore the mechanism of EA regulating ROS and oxidative phosphorylation in DPN.

\section{CONCLUSION}

In conclusion, we used a TMT labeling approach coupled with liquid chromatography with tandem mass spectrometry to show that DPN and EA stimulation drive significant changes in spinal proteins, particularly those involved in oxidative phosphorylation. Our results highlight potential candidate protein biomarkers for DPN diagnosis and treatment.

\section{DATA AVAILABILITY STATEMENT}

The datasets presented in this study can be found in online repositories. The names of the repository/repositories and accession number(s) can be found in the article/ supplementary material.

\section{ETHICS STATEMENT}

The animal study was reviewed and approved by Fujian University of Traditional Chinese Medicine Animal Ethics Committee.

\section{AUTHOR CONTRIBUTIONS}

$\mathrm{XY}$ and $\mathrm{XC}$ made an equal contribution to this research. $\mathrm{ZW}$ and $\mathrm{JT}$ are the co-corresponding authors and they completed the project design, data analysis, and drafted part of the manuscript. All authors contributed to the article and approved the submitted version.

\section{FUNDING}

This study was jointly supported by the National Natural Science Foundation of China $(81774385,81704149)$, the Science and technology platform construction project of Fujian science and Technology Department(Grant No.2018Y2002), the Key Project of "Traditional Chinese Medicine modernization research" in the National Key Research and Development plan (2019YFC1710301). 


\section{REFERENCES}

1. Van H O, Audtin SK, Khan RA, Smith BH, Torrance N. Neuropathic Pain in the General Population: A Systematic Review of Epidemiological Studies. Pain (2014) 155:654-62. doi: 10.1016/j.pain.2013.11.013

2. Lee-Kubli Corinne A CNA. Painful Neuropathy: Mechanisms. Handb Clin Neurol (2014) 126:533-57. doi: 10.1016/B978-0-444-53480-4.00034-5

3. Malisza KL, Jones C, Gruwel MLH, Foreman D, Fernyhough P, Calcutt NA. Functional Magnetic Resonance Imaging of the Spinal Cord During Sensory Stimulation in Diabetic Rats. J Magn Reson Imaging (2009) 30:271-6. doi: $10.1002 /$ jmri.21856

4. Eaton SE, ND H, Rajbhandari SM, Greenwood P, Wilkinson ID, Ward JD, et al. Spinal-Cord Involvement in Diabetic Peripheral Neuropathy. Lancet (2001) 358:35-6. doi: 10.1016/S0140-6736(00)05268-5

5. Shin K-M, Lee S, Lee EY, Kim CH, Kang JW, Lee CK, et al. Electroacupuncture for Painful Diabetic Peripheral Neuropathy: A Multicenter, Randomized, Assessor-Blinded, Controlled Trial. Diabetes Care (2018) 41:e141-2. doi: 10.2337/dc18-1254

6. Bailey A, Wingard D, Allison M, Summers P, Calac D. Acupuncture Treatment of Diabetic Peripheral Neuropathy in an American Indian Community. J Acupunct Meridian Stud (2017) 10(2):90-5. doi: 10.1016/ j.jams.2016.10.004

7. Zhang YJ LF. Effectiveness of Acupuncture for Treatment of Diabetic Peripheral Neuropathy. Med (Baltimore) (2019) 98(39):e17282. doi: 10.1097/MD.0000000000017282

8. Tong Y GH, Han B. Fifteen-Day Acupuncture Treatment Relieves Diabetic Peripheral Neuropathy. J Acupunct Meridian Stud (2010) 3(2):95-103. doi: 10.1016/S2005-2901(10)60018-0

9. Zhang C MY, Yan Y. Clinical Effects of Acupuncture for Diabetic Peripheral Neuropathy. J Tradit Chin Med (2010) 30(1):13-4. doi: 10.1016/s0254-6272 (10)60003-9

10. Feng Y FY, Wang Y, Hao Y. Acupoint Therapy on Diabetes Mellitus and Its Common Chronic Complications: A Review of Its Mechanisms. BioMed Res Int (2018) 22:1-9. doi: 10.1155/2018/3128378

11. Freeman OJ, Unwin RD, Dowsey AW, Begley P, Ali S, Hollywood KA, et al. Metabolic Dysfunction Is Restricted to the Sciatic Nerve in Experimental Diabetic Neuropathy. Diabetes (2016) 65(1):228-38. doi: 10.2337/db15-0835

12. Akude E, Zherebitskaya E, Chowdhury SK, Smith DR, Dobrowsky RT, Fernyhough P. Diminished Superoxide Generation Is Associated With Respiratory Chain Dysfunction and Changes in the Mitochondrial Proteome of Sensory Neurons From Diabetic Rats. Diabetes (2011) 60 (1):288-97. doi: 10.2337/db10-0818

13. Zhang T, Gao Y, Gong Y, Zhou H, Xie P, Guan S, et al. Tang-Luo-Ning Improves Mitochondrial Antioxidase Activity in Dorsal Root Ganglia of Diabetic Rats: A Proteomics Study. BioMed Res Int (2017) 2017:8176089. doi: $10.1155 / 2017 / 8176089$

14. Chowdhury SK DR, Fernyhough P. Nutrient Excess and Altered Mitochondrial Proteome and Function Contribute to Neurodegeneration in Diabetes. Mitochondrion (2011) 11(6):845-54. doi: 10.1016/j.mito.2011. 06.007

15. Shi L, Zhang HH, Xiao Y, Hu J, Xu GY. Electroacupuncture Suppresses Mechanical Allodynia and Nuclear Factor $\kappa$ B Signaling in StreptozotocinInduced Diabetic Rats. CNS Neurosci Ther (2013) 19(2):83-90. doi: 10.1111/ cns. 12035

16. Zhang Z, Shi Y, Cai D, Jin S, Zhu C, Shen Y, et al. Effect of Electroacupuncture at ST36 on the Intestinal Mucosal Mechanical Barrier and Expression of Occludin in a Rat Model of Sepsis. Acupunct Med (2018) 36(5):333-8. doi: 10.1136/acupmed-2016-011187

17. Li J, Wu S, Tang H, Huang W, Wang L, Zhou H, et al. Long-Term Effects of Acupuncture Treatment on Airway Smooth Muscle in a Rat Model of SmokeInduced Chronic Obstructive Pulmonary Disease. Acupunct Med (2016) 34 (2):107-13. doi: 10.1136/acupmed-2014-010674

18. Cao BY, Li R, Tian HH, Ma YJ, Hu XG, Jia N, et al. Pi3k-Glut4 Signal Pathway Associated With Effects of EX-B3 Electroacupuncture on Hyperglycemia and Insulin Resistance of T2DM Rats. Evid Based Complement Alternat Med (2016) 7914387:1-10. doi: 10.1155/2016/7914387

19. Dixon WJ. Efficient Analysis of Experimental Observations. Annu Rev Pharmacol Toxicol (1980) 20:441-62. doi: 10.1146/annurev.pa.20.040180.002301
20. Wang ZF, Li Q, Liu SB, Mi WL, Hu S, Zhao J, et al. Aspirin-Triggered Lipoxin A4 Attenuates Mechanical Allodynia in Association With Inhibiting Spinal JAK2/STAT3 Signaling in Neuropathic Pain in Rats. Neuroscience (2014) 25 (273):65-78. doi: 10.1016/j.neuroscience.2014.04.052

21. Mao-Ying QL, Zhao J, Dong ZQ, Wang J, Yu J, Yan MF, et al. A Rat Model of Bone Cancer Pain Induced by Intra-Tibia Inoculation of Walker 256 Mammary Gland Carcinoma Cells. Biochem Biophys Res Commun (2006) 345(4):1292-8. doi: 10.1016/j.bbrc.2006.04.186

22. Lewis MT, Kasper JD, Bazil JN, Frisbee JC, Wiseman RW. Quantification of Mitochondrial Oxidative Phosphorylation in Metabolic Disease: Application to Type 2 Diabetes. Int J Mol Sci (2019) 20(21):5271-301. doi: 10.3390/ ijms20215271

23. Hunter CA, Kartal F, Koc ZC, Murphy T, Kim JH, Denvir J, et al. Mitochondrial Oxidative Phosphorylation Is Impaired in TALLYHO Mice, a New Obesity and Type 2 Diabetes Animal Model. Int J Biochem Cell Biol (2019) 116(105616):1-25. doi: 10.1016/j.biocel.2019.105616

24. Victor VM, Rocha M, Herance R, Hernandez-Mijares A. Oxidative Stress and Mitochondrial Dysfunction in Type 2 Diabetes. Curr Pharm Des (2011) 17 (36):3947-58. doi: 10.2174/138161211798764915

25. Portt L, Norman G, Clapp C, Greenwood M, Greenwood MT. AntiApoptosisand Cell Survival: A Review. Biochim Biophys Acta (2011) 1813 (1):238-59. doi: 10.1016/j.bbamcr.2010.10.010

26. Ye L, Xiao L, Bai X, Yang SY, Li Y, Chen Y, et al. Spinal MitochondrialDerived ROS Contributes to Remifentanil-Induced Postoperative Hyperalgesia Via Modulating NMDA Receptor in Rats. Neurosci Lett (2016) 10(634):79-86. doi: 10.1016/j.neulet.2016.09.016

27. Zhao WC, Zhang B, Liao MJ, Zhang WX, He WY, Wang HB, et al. Curcumin Ameliorated Diabetic Neuropathy Partially by Inhibition of NADPH Oxidase Mediating Oxidative Stress in the Spinal Cord. Neurosci Lett (2014) 560 (7):81-5. doi: 10.1016/j.neulet.2013.12.019

28. Rasoulian B, Hajializadeh Z, Esmaeili-Mahani S, Rashidipour M, Fatemi I, Kaeidi A. Neuroprotective and Antinociceptive Effects of Rosemary (Rosmarinus Officinalis L.) Extract in Rats With Painful Diabetic Neuropathy. J Physiol Sci (2019) 69(1):57-64. doi: 10.1007/s12576-0180620-x

29. Xu J, Wei X, Gao F, Zhong X, Guo R, Ji Y, et al. Nicotinamide Adenine Dinucleotide Phosphate Oxidase 2-Derived Reactive Oxygen Species Contribute to Long-Term Potentiation of C-Fiber-Evoked Field Potentials in Spinal Dorsal Horn and Persistent Mirror-Image Pain Following HighFrequency Stimulus of the Sciatic Nerve. Pain (2020) 161(4):758-72. doi: $10.1097 /$ j.pain.0000000000001761

30. Schwartz ES, Kim HY, Wang J, Lee I, Klann E, Chung JM, et al. Persistent Pain Is Dependent on Spinal Mitochondrial Antioxidant Levels. J Neurosci (2009) 29(1):159-68. doi: 10.1523/JNEUROSCI.3792-08.2009

31. Ludman T, Melemedjian OK. Bortezomib-Induced Aerobic Glycolysis Contributes to Chemotherapy-Induced Painful Peripheral Neuropathy. Mol Pain (2019) 15:1-17. doi: 10.1177/1744806919837429

32. Ni CM, Sun HP, Xu X, Ling BY, Jin H, Zhang YQ, et al. Spinal P2X7R Contributes to Streptozotocin-Induced Mechanical Allodynia in Mice. J Zhejiang Univ Sci B (2020) 21(2):155-65. doi: 10.1631/jzus.B1900456

33. Munoz FM, Gao R, Tian Y, Henstenburg BA, Barrett JE, Hu H. Neuronal P2X7 Receptor-Induced Reactive Oxygen Species Production Contributes to Nociceptive Behavior in Mice. Sci Rep (2017) 7(1):1-12. doi: 10.1038/s41598017-03813-7

34. Xu J, Chen XM, Zheng BJ, Wang XR. Electroacupuncture Relieves Nerve Injury-Induced Pain Hypersensitivity Via the Inhibition of Spinal P2x7 Receptor-Positive Microglia. Anesth Analg (2016) 122(3):882-92. doi: 10.1213/ANE.0000000000001097

35. Zhong S, Li Z, Huan L, Chen BY. Neurochemical Mechanism of Electroacupuncture: Anti-Injury Effect on Cerebral Function After Focal Cerebral Ischemia in Rats. Evid Based Complement Alternat Med (2009) 6 (1):51-6. doi: 10.1093/ecam/nem062

36. Lu SF, Huang Y, Wang N, Shen WX, Fu SP, Li Q, et al. Cardioprotective Effect of Electroacupuncture Pretreatment on Myocardial Ischemia/Reperfusion Injury Via Antiapoptotic Signaling. Evid Based Complement Alternat Med (2016) 4609784:1-9. doi: 10.1155/2016/4609784

37. Wang C, Liang X, Yu Y, Li Y, Wen X, Liu M. Electroacupuncture Pretreatment Alleviates Myocardial Injury Through Regulating 
Mitochondrial Function. Eur J Med Res (2020) 25(1):1-10. doi: 10.1186/ s40001-020-00431-4

Conflict of Interest: The authors declare that the research was conducted in the absence of any commercial or financial relationships that could be construed as a potential conflict of interest.
Copyright $\odot 2021 \mathrm{Yu}$, Chen, Liu, Jiang, Wang and Tao. This is an open-access article distributed under the terms of the Creative Commons Attribution License (CC BY). The use, distribution or reproduction in other forums is permitted, provided the original author(s) and the copyright owner(s) are credited and that the original publication in this journal is cited, in accordance with accepted academic practice. No use, distribution or reproduction is permitted which does not comply with these terms. 